Trauma Berufskrankh $2003 \cdot 5$ [Suppl 2]: S332-\$339 DOI 10.1007/s10039-002-0649-0
Septische Chirurgie

Matthias Bühler ${ }^{1} \cdot$ Hergo G. K. Schmidt ${ }^{2}$

${ }^{1}$ Abteilung Septische Chirurgie, BG-Unfallklinik Frankfurt am Main

2BG-Unfallkrankenhaus Hamburg

\section{Infektionen nach} Verletzungen am Fuß

\section{Zusammenfassung}

Fußinfektionen nach Trauma können nur selten konservativ behandelt werden. Das Infektionsausmaß ist durch Ultraschall, $\mathrm{Na}$ tivröntgen, CT, MRT und Szintigramm einzugrenzen. Die operative Revision muss die Ursachen der Infektion erkennen und beseitigen, eine evtl. Gelenkbeteiligung therapieren, Sequester und Nekrosen radikal entfernen sowie die Instabilität beheben. Hautweichteildefekte sind sekundär plastisch zu verschließen, Knochendefekte mit autogener Spongiosa aufzubauen.Zur Erreichung rascher Belastungsfähigkeit sind alle physiotherapeutischen Möglichkeiten einschließlich teilentlastendem Gehapparat einzusetzen. Trotz Anwendung aller geeigneten Mittel verbleiben häufig Schädigungen, die eine Berufshilfe und/oder Rentenleistungen erfordern. Um schlechte Ergebnisse bei Infektionen nach Verletzungen am Fuß zu vermeiden, muss in erster Linie der Patient mit seinen individuellen Bedürfnissen behandelt werden. Oft sind Etappenrevisionen erforderlich, denn bekanntermaßen verhelfen viele kleine Schritte dazu, große Probleme zu lösen.

\section{Schlüsselwörter}

Fußinfektionen nach Trauma - Diagnostik bei Fußinfektionen - Therapie von Infektionen nach Verletzungen am Fuß ach Verletzungen am Fuß mit Durchtrennung des intakten, schützenden Hautmantels kann es auf unterschiedlichen Wegen zur Infektion von Weichteilen oder Knochenstrukturen kommen. Zum einen kann eine hohe primäre Keiminokulation durch erfolgte Traumatisierung, also als unmittelbare Verletzungsfolge, zur Infektion führen, zum anderen kann eine sprunghafte bakterielle Vermehrung in traumatisch entstandenen Weichteil- und Knochennekrosen eintreten, welche sekundär den Behandlungsverlauf kompliziert. Darüber hinaus besteht die Möglichkeit einer Infektauslösung durch die Einbringung von Fremdmaterial und Bakterien während der operativen Behandlung.

Der Fuß ist besonders infektionsgefährdet, weil hier nur eine dünne Weichteilabdeckung besteht und auch beim Gesunden die Durchblutung dort deutlich schlechter ist als in proximal gelegenen Extremitätenabschnitten. Es ist bekannt, dass die Durchblutungsrate am Fuß 4-mal schlechter ist als beispielsweise in der Umgebung des Hüftgelenks, an den oberen Extremitäten oder am Schädel. Darüber hinaus manifestieren sich gerade am Fuß viele Symptome anderer Erkrankungen besonders ungünstig, wie z. B. bei Diabetes mellitus, dem postthrombotischen Syndrom oder der arteriellen Verschlusskrankheit. Die genannten Gründe führen zu einer deutlich höheren Infektionsrate nach Fußverletzungen, im Vergleich zu Verletzungen in weiter proximal gelegenen
Abschnitten. Die postoperative Infektrate ist am Fuß höher als an der oberen Extremität.

\section{Diagnostik}

\section{Klinische Diagnostik}

Nach konservativer oder operativer Behandlung einer geschlossenen oder offenen Fraktur ist der ungestörte komplikationslose Heilverlauf durch kontinuierlich abnehmende Schwellung in Verbindung mit eintretender Schmerzreduktion im Verlauf von etwa 7 Tagen gekennzeichnet. Kommt es zu einer Infektion, bilden sich die posttraumatischen Symptome dagegen nicht kontinuierlich zurück, es treten vielmehr weitere Symptome hinzu. Es kommt zu den klassischen Infektzeichen wie Rötung (rubor) und Überwärmung (calor). Dabei entwickeln sich meist Fieber sowie, beim Auftreten eines Abszesses, eine fluktuierende Schwellung im Verletzungsbereich. Bei phlegmonöser Ausbreitung findet sich eine zunehmende

(c) Springer-Verlag 2003

Dr. Matthias N. Bühler Abteilung Septische Chirurgie, BG-Unfallklinik Frankfurt am Main, Friedberger Landstraße 430, 60389 Frankfurt am Main, E-Mail: BuehlerSC@Hotmail.com, Phone: 069-4752022, Fax: 069-4752018 


\section{Bühler ·H.G.K.Schmidt}

\section{Infections after foot injuries}

\section{Abstract}

When trauma is followed by infections in the foot it is rarely possible to treat these adequately by conservative means. After clinical examination, both laboratory tests and microbiological analysis are mandatory. The severity and extent of the infection must be determined by ultrasonography, plain X-rays, $\mathrm{CT}, \mathrm{MRI}$ and scintigraphy. The causes of the infection must first be found and then removed by specific surgical revision techniques: any joint involvement must be treated, instability eliminated, and radical debridement performed to remove areas of soft tissue necrosis and bone sequestra. Skin and soft tissue defects need to be closed by plastic reconstruction performed as a secondary procedure; bony defects can be built up with autogenous cancellous bone. All the options open to physiotherapy, including appliances allowing partial weight-bearing, should be used with the aim of early mobilisation and weight-bearing. Even so, impairments that are sufficiently severe to require occupational rehabilitation and/or justify a partial disability pension often persist. To avoid poor results of treatment in the case of infections following injury to the foot, it is essential that the primary treatment be individually tailored to the patient. Frequent revision is needed at each stage, since many small steps can help to solve big problems.

\section{Keywords}

Posttraumatic foot infections · Diagnosis · Treatment schmerzhafte Hautrötung mit Schwellung, wobei Spannungsblasen der Haut auftreten können.

Wird die Infektion nicht rechtzeitig erkannt, die Behandlung nicht konsequent durchgeführt, besteht die Möglichkeit der Fistelentstehung mit seröser, später eitriger Sekretion. Auch infektbedingte Knochen- und Weichgewebenekrosen können entstehen. Unbehandelt kann die Infektion vom Fuß ausgehend metastatisch Infektionen in anderen Extremitätenabschnitten oder Organen erzeugen. Die Infektion kann sich aber auch kontinuierlich oder diskontinuierlich nach zentral hin über das Subkutangewebe, die Faszien sowie die Lymphbahnen ausbreiten. Auf diesem Weg entstehen eine Fasziitis, eine Lymphangitis oder eine Lymphadenitis. Schließlich kann es bei unzureichender oder zu spät eingeleiteter Therapie einer Fußinfektion zur generalisierten Infektion, also zur Sepsis mit Multiorganversagen kommen, der Tod kann eintreten.

\section{Laboruntersuchungen, mikrobiologische Diagnostik}

Im posttraumatischen komplikationslosen Verlauf sind sowohl die BSG beschleunigt als auch die Leukozytenzahl sowie das CRP erhöht. Entwickelt sich eine Infektion, kommt es nicht zu einer Normalisierung der genannten Parameter, sie steigen vielmehr in ungewöhnlicher Weise sehr hoch an oder zeigen nach kurzzeitiger Rückbildung über wenige Tage einen erneuten Anstieg. Trotz persistierender Infektion können die genannten Parameter im weiteren Verlauf, also nach Wochen und Monaten, wieder Normalwerte aufweisen, ohne dass die Infektion saniert wurde, $d . h$. normale Laborwerte schließen eine Infektion nicht aus. Die Beurteilung der Laborwerte hat immer in Verbindung mit der vorliegenden klinischen Situation zu erfolgen.

Nach entstandener oder bei sich entwickelnder Infektion ist es zwingend erforderlich, die Art und speziell die Resistenz des infektauslösenden Erregers zu kennen, d. h. ein mikrobiologisches Abradat wird benötigt. Eiter ist häufig steril, es ist daher günstiger, Gewebe vom Rand der Infektion zu gewinnen. Das gewonnene Abradat von infiziertem Gewebe, entnommen mit Skalpell, LuerZange oder Kürettenlöffel, muss mög- lichst rasch, in einem geeigneten Transportmedium eingebettet, zur bakteriologischen Untersuchung gebracht werden. Ist bereits eine antibiotische Therapie eingeleitet, kann die mikrobiologische Untersuchung oft keinen Keimnachweis führen. Diese Tatsache muss unbedingt beachtet werden. Nach Einleiten der Antibiotikatherapie ist der Nachweis des infektauslösenden Erregers also erheblich erschwert, es kommt zu falscher Beurteilung oder gar zu Fehlbestimmungen. Ein mikrobiologisches Ergebnis unter laufender oder gerade eingestellter Antibiotikatherapie von weniger als 2 Tagen, welches ,kein Keimnachweis" lautet, bedeutet keinesfalls, dass keine bakteriologische Infektion vorliegt.

\section{Bild gebende Verfahren}

\section{Sonographie}

Im Frühstadium einer sich entwickelnden Infektion ist sonographisch gelegentlich eine Schwellung und/oder eine Flüssigkeitsansammlung im therapierten Verletzungsbereich nachweisbar. Auch primär nicht betroffene Strukturen können sonographische Veränderungen aufweisen.

\section{Röntgen}

Das Nativröntgen zeigt anfangs keine gravierenden infektspezifischen Veränderungen. Etwa 10 Tage nach dem Trauma sind außer einer Weichgewebeschwellung oder ggf. einem Gelenkerguss keine wesentlichen Veränderungen zu finden, welche auf eine Infektion hinweisen. Bei Fortbestehen der Infektion kann es dann zu Veränderungen der Knochenbälkchenstruktur mit röntgenologisch nachweisbaren Lysen, zur fleckförmigen Auflockerung der kortikalen Struktur mit einliegenden Lysezonen kommen. Die Frakturlinien bleiben auch im Infekt in der Regel als solche scharf erhalten und werden nicht verwaschen. Schließlich findet sich eine scharfe Abgrenzung erkrankter Knochenbezirke von ihrer Umgebung. Nicht durchblutete Areale, also Knochennekrosen oder auch Sequester, bilden sich zur immer strahlendurchlässigeren Umgebung hin deutlich ab. Infizierte Gelenke zeigen anfangs Ergüsse bei erhaltenen Strukturen. Kommt es zur na- 
tivröntgenologischen Darstellung durchlöcherter subchondraler Zonen, liegt bereits eine fortgeschrittene Gelenkzerstörung vor.

\section{Computertomographie}

Im CT sind die infektbedingten Veränderungen etwas früher als im Nativröntgen erkennbar und gelegentlich auch besser abgrenzbar.

\section{Kernspintomographie}

Das MRT kann Veränderungen der Weichteilstrukturen darstellen, die Differenzierung der gefundenen Veränderungen ist $z$. T. jedoch schwierig, das Ausmaß einer Entzündung wird oft falsch eingeschätzt. Eine enge Rückkopplung zwischen dem behandelnden Arzt und dem Radiologen ist hier sehr wichtig, um den erhobenen Bild gebenden Befund in korrekte Beziehung zum aktuellen klinischen Befund setzen zu können. Die Abgrenzung zwischen „normalem Heilverlauf“ und "pathologischem Heilverlauf“ ist schwierig.

\section{3-Phasen-Skelettszintigramm}

Das 3-Phasen-Skelettszintigramm mit Technetium zeigt nach $24-48$ h eine deutliche Mehrbelegung bereits in der 1. und 2. Phase. Leider zeigt das Szintigramm auch bei ungestörtem Heilverlauf eine auffällige Mehrbelegung, die z. T. über Jahre hinaus bestehen bleiben kann. Das Skelettszintigramm ist nur bei mehrfacher Durchführung aussagekräftig, Verlaufsbeobachtungen sollten durchgeführt werden, um eine Infektion nachweisen bzw. ausschließen zu können.

\section{Invasive Diagnostik}

Bei unklarer Symptomatik und vorliegender Fistel kann eine Kontrastmitteluntersuchung, also eine röntgenologische Fisteldarstellung, erfolgen. Phlebographische Untersuchungen zum Ausschluss einer Thrombose sowie eine Angiographie der entsprechenden Extremität können differenzialdiagnostische Fragen klären.

Muss bei vorangeschrittener Infektion die Entscheidung „Erhalt oder Amputation der Extremität" getroffen werden, ist die arterielle Durchblutungssi- tuation unbedingt zuvor abzuklären. Nach angiologischer Untersuchung und Dopplersonographie können ein AngioMRT oder eine Arteriographie durchgeführt werden.

\section{Intraoperative Diagnostik}

Ist eine operative Versorgung der Infektion am Fuß erforderlich, muss intraoperativ die Möglichkeit bestehen, eine Durchleuchtung durchzuführen oder Röntgennativbilder in 2 Ebenen anzufertigen. Dies kann beim Auffinden kleiner Infektionsherde oder auch zum Festlegen des Ausmaßes der erforderlichen Sequestrektomie hilfreich sein, auch zur Stellungskontrolle sowie zum Nachweis der erfolgten vollständigen Metallentfernung ist die intraoperative Röntgenuntersuchung anzuwenden.

\section{Typische Begleitverletzungen}

Am Fuß sind die Knochendimensionen begrenzt, häufig ist der Knochen von mehreren Gelenken umgeben oder flankiert. An den Knochen grenzen oft Sehnen oder Sehnenscheiden, häufiger jedoch Bänder. Die genannten Strukturen halten das Voranschreiten einer Infektion nicht auf, deshalb sind nach Fraktur eines Fußknochens häufig bereits nach kurzer Zeit die angrenzenden Gelenke und Weichteilstrukturen in die Infektion mit einbezogen. Eine Behandlung einer Fuß-/Knocheninfektion kann nur dann erfolgreich sein, wenn gleichzeitig die beteiligten Strukturen, also Gelenke, Bänder, Faszien, Sehnen und Muskeln, mitbehandelt werden. Dies sollte bei der präoperativen umfassenden Diagnostik beachtet werden, die präoperative Nutzung von CT und/oder MRT wird dabei empfohlen.

\section{Klassifikation}

Eine allgemein gültige Klassifikation der Fußinfektionen steht nicht zur Verfügung.

\section{Konservative Therapie}

\section{Therapieziel}

Die Infektion soll beruhigt bzw. beseitigt, die Funktion des Fußes wieder hergestellt werden.

\section{Indikation}

Das Therapieprinzip bei Infektionen nach Verletzungen am Fuß ist der frühestmögliche Behandlungsbeginn. Ob zunächst konservativ oder aber sofort operativ behandelt werden muss, ist individuell zu entscheiden. Es ist durchaus gestattet, bei eingegrenzter, also begrenzter, Infektion - und nur bei dieser einen konservativen Behandlungsversuch zu unternehmen.

Der Begriff „begrenzte Infektion“ kann erst dann Anwendung finden, wenn durch eine umfassende Diagnostik die Ausdehnung der Infektion sicher geklärt wurde. Nach operativ behandelter Fraktur am Fuß betrifft die Infektion in der Regel das gesamte Operationsgebiet.

\section{Kontraindikation}

Infektionen nach offen gewesener und/ oder operativ behandelter Fraktur müssen insbesondere im Mittel- und Rückfußbereich bei auftretender Infektion operativ revidiert werden. Eine Ausnahme können hier Kirschner-Draht-Osteosynthesen bilden.

\section{Therapiemaßnahmen}

Als konservative Behandlungsmaßnahme kann eine lokale Behandlung mit

- Hochlagerung,

- Ruhigstellung in Funktionsstellung,

- täglichen Fußbädern oder Wundabduschungen sowie

- intermittierenden Kälteapplikationen

empfohlen werden.

Die Antibiotikatherapie erfolgt möglichst gezielt nach Antibiogramm. Sie muss ausreichend dosiert sein. Anfangs wird dabei i. v., bei Rückgang der Infektionszeichen oral therapiert. Ist der Erreger nicht oder noch nicht bekannt, wird mit einem breiten Betalaktamantibiotikum oder Zephalosporin oder Chinolon behandelt. Nach abgeschlossener mikrobiologischer Untersuchung und vorliegendem Antibiogramm muss die Antibiotikatherapie ggf. entsprechend geändert werden. Die Dauer der Antibiotikatherapie richtet sich nach dem Verlauf, empfohlen wird eine Therapie für mindestens 3 Tage über den Rückgang der Symptomatik hinaus. Tägliche Wundinspektion mit Verbandswechsel 
ist obligat, wobei sich desinfizierende Salben (z. B. Betaisodona) oder antiseptische Gele (z. B. Lavaseptgel o,2\%) in Verbindung mit Salbengaze bewährt haben. Tägliche Fußbäder in Betaisodonalösung oder auch in Kamille sind ebenso heilungsfördernd wie Ganzkörperoder Duschbäder, welche einer Keimreduktion dienen. Liegt eine abklingende Infektsymptomatik vor, sollte eine frühfunktionelle Mobilisationsbehandlung durch den erfahrenen Physiotherapeuten begonnen werden.

\section{Therapieentscheidungen}

Die jeweilige Therapieentscheidung über

- Dauer der Antibiose,

- Art des Verbands,

- Beginn der Mobilisation und

- Änderung des Behandlungsverfahrens

richtet sich am klinischen Verlauf aus, wobei die Beurteilung möglichst von ein und demselben Arzt vorgenommen werden sollte. Ist dies nicht möglich, sind eine exakte und umfassende Dokumentation oder anderweitige Befundübermittlung unumgänglich.

\section{Fehler, Gefahren und Komplikationen}

Die wesentliche Gefahr der konservativen Therapie besteht in der Verschleierung der Symptomatik sowie in einem Wechsel von der akuten in die chronisch schleichende Verlaufsform. Die konservative Behandlung einer Fußinfektion ist nur so lange gestattet, wie sich die Infektsymptomatik sowohl subjektiv als auch objektiv kontinuierlich zurückbildet. Entscheidende Kriterien sind neben laborchemischen Untersuchungen und klinischer Verlaufskontrolle das Empfinden des Patienten. Diesem subjektiven Faktor ist die wesentliche Bedeutung zuzumessen.

Bilden sich die Symptome zurück, normalisieren sich CRP und - immer verzögert - auch die BSG, kann der Patient problemlos an der Mobilisationsbehandlung teilnehmen, die konservative Therapie kann fortgesetzt werden. Fehlt jedoch 1 der 3 diagnostischen Säulen oder besteht unverändert Schmerz, der nicht auf die Verletzung zurückgeführt werden kann, muss operativ vorgegangen werden.
Eine weitere Gefahr stellt die unzureichende antibiotische Therapie dar. Einerseits kann das gewählte Antibiotikum gar nicht oder nur sehr eingeschränkt wirksam sein - trotzdem ergibt sich zumindest anfangs oder vorübergehend eine Besserung - andererseits kann das Antibiotikum nach anfänglich guter Wirkung, mehr oder minder plötzlich im Verlauf, keine Wirkung mehr zeigen. In diesem Fall ist unbedingt abzuklären, ob die Infektion zu Recht konservativ behandelt wird (Infektausdehnung, beteiligte Strukturen, Instabilität?). Ergibt sich weiterhin die Berechtigung zur konservativen Therapie, muss abgeklärt werden, ob es z. B. zum Erregerwechsel oder zum Auftreten einer Resistenz gekommen ist. Eine erneute bakteriologische Diagnostik ist in diesem Fall auch bei laufender, nicht geänderter Antibiose erforderlich.

\section{Operative Therapie und Operationstechnik}

\section{Patientenaufklärung}

Bei erforderlicher Revision einer Infektion, nach offener oder geschlossener und/oder operativ behandelter Fraktur am Fuß, muss die Aufklärung alle evtl. eintretenden operativen Möglichkeiten einschließen. Insbesondere ist im $\mathrm{Ge}$ spräch mit dem Patienten auf die Gefahren einer Metallentfernung im Infekt einzugehen. Darüber hinaus muss dargestellt werden, dass bei Instabilität evtl. eine externe Osteosynthese durchgeführt werden muss, dass ausgedehnte Knochen- und/oder Weichteilverluste auftreten können, dass die Infektion trotz operativer Behandlung weiter bestehen kann, dass mehrere Folgeeingriffe zur Infektberuhigung und zum anschließenden Defektaufbau erforderlich werden und bei Erfolglosigkeit der Therapie ggf. die Amputation notwendig werden könnte. Die Gefahren von Gefäß-, Nerven- und Sehnenverletzungen oder Verlusten sind ebenso darzustellen, wie vermutlich verbleibende Funktionseinbußen.

Sollte die umfassende Aufklärung mit Darstellung der evtl. erforderlichen Maßnahmen den Patienten verunsichern oder ihm unzureichend erscheinen, ist es angezeigt, dem Patienten die Möglichkeit einzuräumen, eine 2. externe Spezialistenmeinung einzuholen.

\section{Operationsvorbereitung}

Das Ausmaß der geplanten Revision sollte möglichst exakt eingegrenzt werden, eine umfassende präoperative Diagnostik ist dabei unbedingt erforderlich. Der infektauslösende Keim sollte bekannt sein, dadurch ist die Wahl des geeigneten Antibiotikums möglich, außerdem kann dadurch geklärt werden, ob ggf.lokale Antibiotikaträger Anwendung finden können. Festgelegt werden müssen der Operationszeitpunkt, der geeignete Operateur und das erforderliche Instrumentarium.

Ist der infektauslösende Erreger bekannt, sollte die Antibiotikatherapie bereits präoperativ in ausreichender Dosierung begonnen werden. Ist der Erreger noch nicht bekannt, sollten in jedem Fall intraoperativ Gewebeproben aus den Randbezirken der Infektion für die bakteriologische Untersuchung gewonnen werden.

\section{Instrumentarium}

Außer den gängigen Normalsieben ist das Instrumentarium zur Materialentfernung bereitzustellen, evtl. müssen auch unbekannte Materialien entfernt werden. Jedenfalls müssen Schraubendreher und Schraubenschlüssel verschiedener Größe, unterschiedliche Zangen, Entfernungsset für Krampen, Blount-Klammern usw. verfügbar sein. Darüber hinaus muss die Möglichkeit der externen Stabilisation gegeben sein, d. h. unterschiedliche Fixateur-externeSysteme müssen verfügbar sein (AO-Fixateur, Hoffman-II-Fixateur, Ringfixateur). Auch die Jet-Lavage-Druckspülung inklusive Ringer-Lösung müssen einsatzbereit sein. Zur postoperativen Wundabdeckung wird geeignetes Verbandmaterial benötigt, Hautersatz, Coldex-Verband, Vakuumokklusionsverband, Salbengaze, Betaisodonasalbe und Lavaseptgel können erforderlich werden.

Zur postoperativen Ruhigstellung oder auch als Spitzfußprophylaxe ist evtl. das Anlegen eines Gipsverbands notwendig. Intraoperativ sollten die Möglichkeit der Bildwandleruntersuchung bzw. Dokumentation oder aber die Durchführung von Röntgenaufnahmen möglich sein. 


\section{Septische Chirurgie}
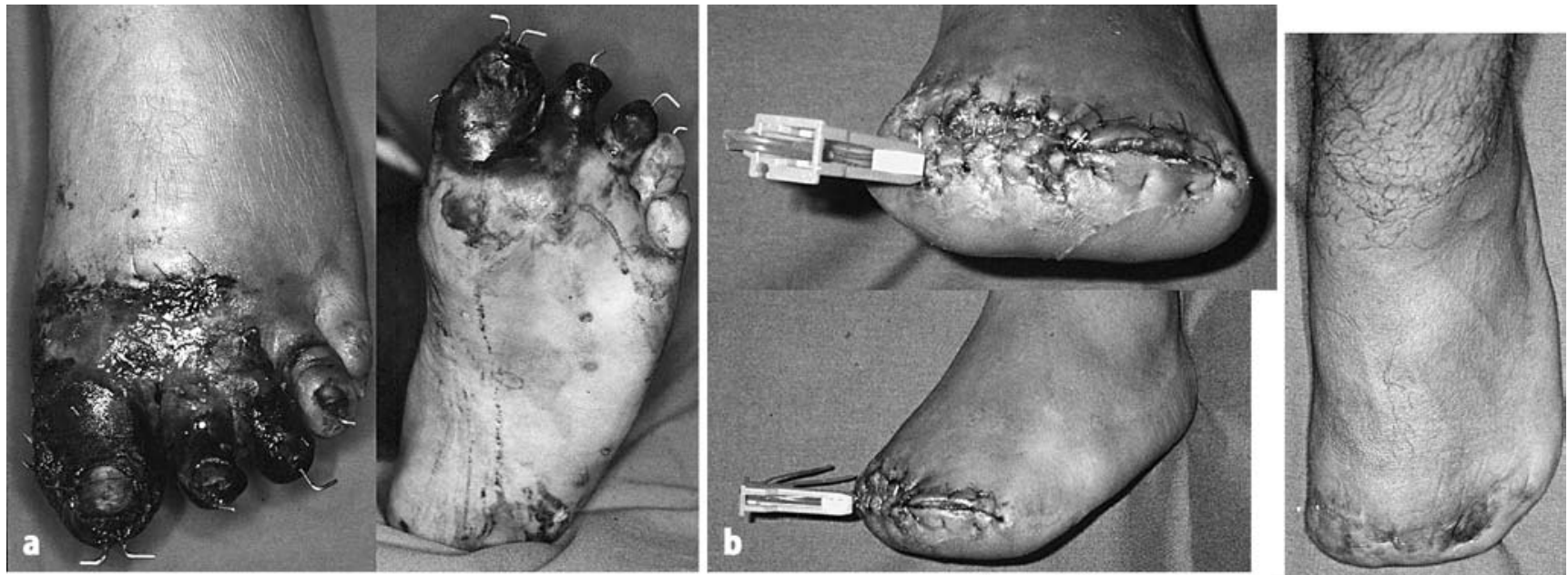

Abb. 1a-c $\Delta$ Vorfußquetschung eines 19-jährigen Manns mit Frakturen der Zehen 1-4, a Aufnahmebefund nach Vorfußerhaltungsversuch, $b$ Grenzzonenamputation in Höhe der Zehengrundgelenke; aufgrund des fehlenden Weichteilmantels kein primärer Wundverschluss möglich; kontinuierliche Wundranddehnung (Dermatotraktion) unter täglicher offener Wundbehandlung; c nach abgeschlossener Dermatotraktion Sekundärnaht, Abschlussbild vor Entlassung aus stationärer Behandlung: bei stabiler Weichgewebesituation Vollbelastung im Konfektionsschuh möglich

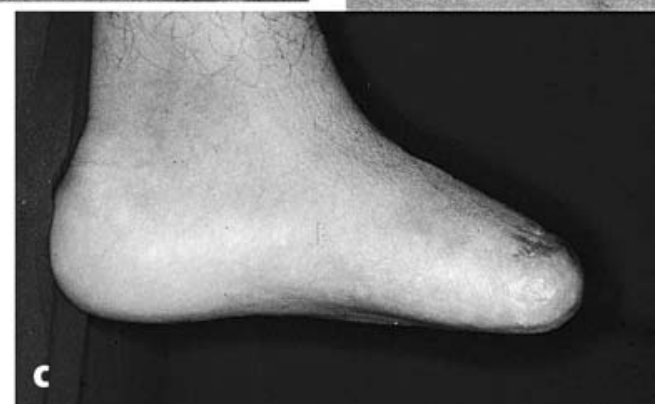

\section{Anästhesie und Lagerung}

Die operativen Eingriffe bei Infektionen am Fuß sollten in Allgemeinnarkose oder rückenmarknaher Analgesie erfolgen. Die Lagerung erfolgt meist auf dem Rücken, nur in manchen Fällen der Fersenbeininfektion mit Plattenosteosynthese von lateral sind die Seiten-, in seltenen Fällen auch die Bauchlage günstiger.

\section{Operationstechnik}

Die Operation ist in erster Linie von der vorgegebenen Situation abhängig: Ziel der Behandlung sind grundsätzlich das Aufdecken der Infektionsursache sowie deren Beseitigung. Liegt keine interne Osteosynthese ein, bestehen keine Nekrosen und/oder sequestrierte Knochenareale, kann es sich um eine Gelenkinfektion, Sehnenscheideninfektion oder Hohlfußinfektion handeln, wobei z. B. die Plantaraponeurose nekrotisch sein kann. Bei wesentlichen Begleiterkrankungen, wie insulinpflichtigem Diabetes mellitus und/oder pAVK, ist grundsätzlich präoperativ zu prüfen, wie weit eine Erhaltung des infizierten Fußes möglich ist. Grundsätzlich steht jedoch fest, dass für den erfahrenen Infektoperateur viel mehr zu erhalten ist als sich der unerfahrene Operateur überhaupt vorstellen kann (Abb. 1)!

Die Infektchirurgie am Fuß ist um so schwieriger, je früher sie erfolgt, d. h. bei sehr rascher früher Revision ist das evtl. Ausmaß der infektbedingten Zerstörung am schwierigsten abzugrenzen, andererseits entlastet die frühe Revision die betroffenen Strukturen am schnellsten und vermeidet weitere Schäden. Bei geschlossenen Weichteilen und eingetretener Infektion ist es häufig günstig, rasch zu revidieren, damit offene Verhältnisse zu schaffen und Nekrosen sowie eindeutige Sequester zu entfernen. Des Weiteren ist eine Spülung erforderlich, lokale Antibiotikaträger werden eingelegt, ggf. wird offen weiterbehandelt. In programmierten Etappenrevisionen, beginnend mit dem geplanten Secondlook-Eingriff etwa 4-10 Tage später, werden das definitive Débridement, die Sequestrektomie sowie die Nekrosektomie ausgeführt, wobei die Abgrenzung zu diesem Zeitpunkt meist deutlich einfacher und zutreffender gelingt (Abb. 2).

Bei der Aufklärung des Patienten ist das etappenweise Vorgehen unbedingt zu besprechen.

Hinsichtlich der einzelnen Verletzungen sollten folgende Maßnahmen gewählt werden:
Infizierte Fraktur ohne Osteosynthese und ohne grobe Instabilität

Hier handelt es sich in der Regel um ein umschriebenes Infektionsproblem, welches operativ gelöst und akribisch ausgeräumt werden muss.

- Cave - Angrenzende Gelenkinfektionen dürfen nicht übersehen werden. Ebenso muss auf eine gleichzeitige Sehneninfektion und/oder Instabilität geachtet werden, die sowohl unfallbedingt, aber auch durch eine erfolgte Revision entstanden sein kann. Bei geringer Instabilität und fehlender Begleiterkrankung kann die Ruhigstellung im Gips ausreichend sein, bei grober Instabilität und/oder bei Begleiterkrankungen wie Diabetes mellitus, pAVK oder Nervenerkrankung, ist eine externe Osteosynthese dringend anzuraten, weil ansonsten die vorliegende Problematik nicht gebessert wird.

\section{Infizierte Fraktur mit Defekt und/oder grober Instabilität}

Auch hier muss sehr gründlich débridiert werden. Es kommen die Jet-Lavage-Druckspülung oder eine mehrfache intensive Spülung mit 6oer-Spritze zum 

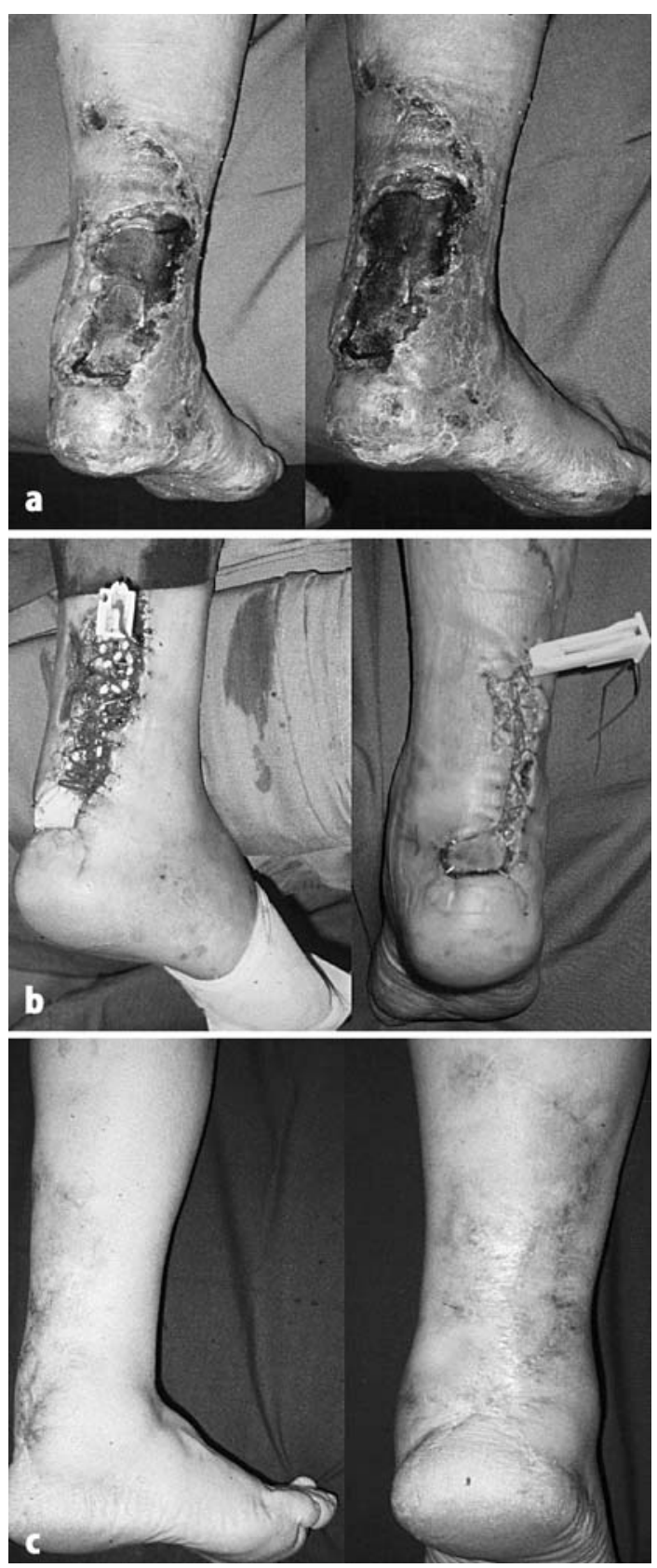

Abb. $2<$ a Klinische Ausgangssituation bei Behandlungsübernahme eines 53-jährigen Patienten: Zustand nach Fersenbeinfraktur 3 Monate zuvor mit posttraumatischer 0 steitis. Der fersennahe Weichgewebedefekt war mit Suralislappenplastik verschlossen worden, es kam jedoch zu einer Lappennekrose bei persistierender Fersenbeinosteitis. $b$ Klinisches Bild nach Nekrosektomie und Sequesterektomie, dynamische Wundranddehnung, passagere Defektdeckung, lokale Antibiotikatherapie (links 1. postoperativer Tag; rechts 14. postoperativer Tag). c Endbefund nach abgeheilter Sekundärnaht, beruhigte Fersenbeinosteitis

Einsatz, angrenzende Gelenkinfektionen müssen dabei radikal ausgeräumt werden. Die kleinen Fußgelenke zeigen meist nur wenig Synovialishypertrophie. Bei einer Infektion kommt es oft rasch zur Ablösung von Knorpel, welcher intraoperativ mit dem Elevatorium abgehoben werden kann. Zeigt sich diese intraoperative Situation, muss der Gelenkknorpel komplett entfernt werden, verbliebene Reste führen zu einer Infektpersistenz. Sequestrierte Knochenanteile sind radikal zu entfernen, auch infizierte Spongiosa muss vollständig ausgeräumt werden, und zwar so weit, bis kein Granulationsgewebe mehr zwischen den Knochenbälkchen nachweisbar ist. Die Sequestrektomie muss radikal und vollständig erfolgen, dabei darf nicht an den späteren Defektaufbau gedacht werden. Die Infektberuhigung kann nur dann gelingen, wenn man vor dem entstehenden Defekt keinen Respekt hat.

Nach radikalem Débridement, nach ausgiebiger Spülung und begleitender systemischer Antibiotikatherapie hat sich die Implantation von lokalen Antibiotikaträgern zum Erreichen der Infektberuhigung bewährt. Da nach Se- questrektomie häufig Knochendefekte resultieren, welche sekundär aufgefüllt werden müssen, kann mittelfristig die passagere Implantation von Septopal erfolgen. Bei entsprechendem Antibiogramm können auch Copal ${ }^{\circledR}$ (Glindamycin-Gentamycin-PMMA-Kugeln) bzw. Vancomycin-Gentamycin-PMMAKugeln implantiert werden, welche auf spezielle Bestellung bei der Fa. Biomet Merck angefordert oder selbst gefertigt werden können. Die genannten Kugelketten dienen der lokalantibiotischen Therapie und werden vor Durchführung des Defektaufbaus als Platzhalter genutzt.

Zur Beseitigung der Instabilität ist eine externe Osteosynthese erforderlich. Am Fuß können Knochenschrauben gut im Fersenbein und Talus, mit endständigem oder auch mittelständigem $\mathrm{Ge}$ winde - an MFK-I und MFK-V jeweils mit endständigem Gewinde - platziert werden. Auch Hybridmontagen, also eine Kombination von Ringfixateur und Hoffmann-II-Fixateur, können zur Fußruhigstellung genutzt werden. Meist müssen das untere und/oder obere Sprunggelenk mit einer stabilisierenden Methode überbrückt werden. Der Ringfixateur ist für die Fußstabilisierung noch besser geeignet als die Klammerund die Rahmenfixateure. Hierbei wird mit einfachen, doppelten und gekreuzten Kirschner-Drähten gearbeitet, die in aller Regel, insbesondere bei Verwendung von Kirschner-Drähten mit Stopperoliven, sehr schonend und trotzdem extrem stabil, auf Dauer platziert werden können. Auch die Standzeit eines Ringfixateurs ist viel höher als die eines Klammer- und Rahmenfixateurs, die Stabilität ist deutlich höher.

\section{Infizierte Fraktur mit einliegender interner Osteosynthese}

Schwer wiegende Infektionen können sich natürlich nach jeder Osteosynthese entwickeln, dies hängt sehr von den Begleitumständen, den Begleitverletzungen sowie den Begleiterkrankungen ab. Stabil einliegende Kirschner-Drähte können, je nach lokalem Befund, auch in der Infektion belassen werden, so lange sie eine sichere Stabilität erzeugen, Fehlstellungen vermeiden und korrekt platziert sind, was intraoperativ genauestens mit dem Bildwandler zu überprüfen ist. Infizierte Plattenosteosynthesen können 


\section{Septische Chirurgie}

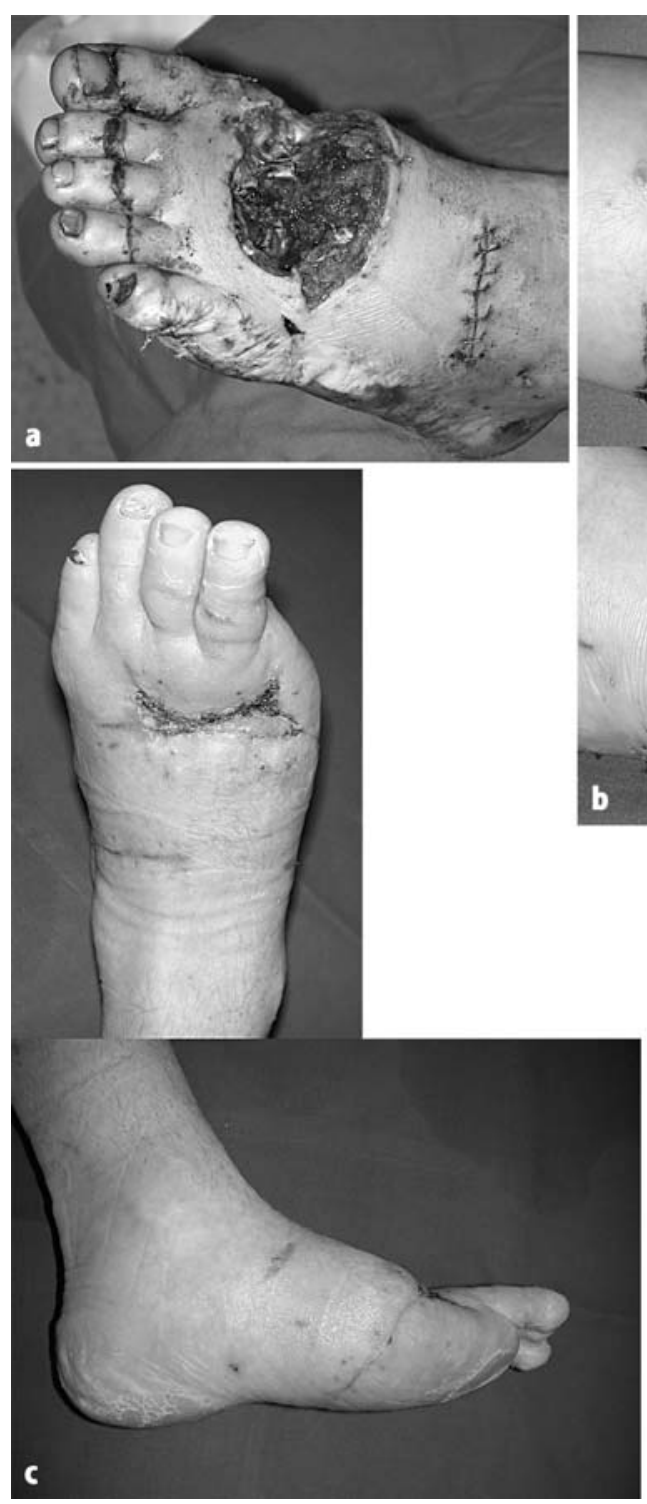

Abb. 3a-c $\Delta$ Subtotale Vorfußamputation nach Ackerfräsenverletzung, a Zustand nach Erstversorgung, b Großzehenamputation nach arterieller Thrombosierung, temporärer Hautverschluss nach erfolgter Etappenrevision mit lokaler Antibiotikatherapie, c Befund vor Entlassung des 21-jährigen Patienten in sein Heimatland

bei der Frühinfektion und stabilem korrektem Materialsitz zunächst einer Frühintervention unterzogen werden, mit dem Ziel des Osteosyntheseerhalts.

Kommt es durch diesen Rettungsversuch nicht zu der erhofften Infektionsberuhigung, also einer Besserung der Infektionssymptomatik nach etwa 5 bis maximal 10 Tagen, oder aber besteht primär keine Chance des Implantaterhalts, ist die Metallentfernung vorzunehmen. Häufig wird die Problematik erst nach Entfernen der Platte richtig sichtbar, weil jetzt die Sequester zu Tage treten. Die Lage der Schrauben ist zu- nächst kritisch unter Bildwandler zu überprüfen, ragen die Schrauben in angrenzende Gelenke, kann auch hier eine Infektion bestehen, die Entfernung des Materials und die jeweilige Gelenkrevision werden erforderlich. Bei einer Kalkaneusinfektion mit liegender Platte ist die Infektion meist viel ausgedehnter als erwartet, oft muss viel vom Fersenbeinknochen entfernt werden, nicht selten ist auch das untere Sprunggelenk in die Infektion mit einbezogen. Nach Entfernen der Platte und des infizierten bzw. sequestrierten Knochens ist zu entscheiden,
- ob eine externe Osteosynthese notwendig ist,

- ob angrenzende Gelenke infiziert sind (diese müssen dann ausgeräumt werden) oder

- ob Weichteile nekrotisch sind und einer Exzidierung zugeführt werden müssen.

\section{Gelenkinfektionen, Weichteilnekrosen, Hautdefekte}

Wie bereits mehrfach erwähnt, müssen infizierte Gelenke am Fuß radikal ausgeräumt werden, sie erholen sich meist nicht von ihrer Infektion. Ein Erhalt ist nur bei sehr frühem Eingreifen und evtl. bei Kindern möglich. Weichteilnekrosen, insbesondere von Faszien und Bändern, sind komplett zu entfernen, nekrotische Muskulatur - sie sieht wie gekochtes Gewebe aus - ihr Entfernen ist mit dem scharfen Löffel einfach möglich - ist radikal auszuräumen, infizierte Sehnenscheiden müssen komplett entfernt werden, ebenso nekrotische Sehnen.

Hautdefekte werden primär mit Hautersatzmaterialien (Epigard ${ }^{\circledR}$, Cutinova ${ }^{\circledR}$ usw.) gedeckt (Abb. 3), je nach Befund ist ggf. eine Konditionierung mit Vakuumokklusionsverband möglich. Im Bereich der Ferse, der Fußsohle sowie des Rückfußes sind fast regelmäßig sekundär plastische Maßnahmen erforderlich.

\section{Postoperative Behandlung}

Die postoperative Therapie beinhaltet die Fortsetzung der systematischen Antibiotikatherapie, welche nach Zeichen des Infektrückgangs von i. v. auf oral umgesetzt wird. Es erfolgen eine Ruhigstellung, die Hochlagerung und tägliche Verbandswechsel, evtl. mit Fuß- oder Ganzkörperbad. Wichtig ist die kritische Beurteilung des weiteren klinischen Verlaufs unter regelmäßigen Labor- und Röntgenkontrollen, ggf. ergänzt durch Szintigraphie, CT- und MRT-Diagnostik. Bei Ruhigstellung der infizierten Extremität sollte ein Mobilisationsprogramm für den sonstigen Organismus unter $\mathrm{Zu}$ hilfenahme von Physiotherapie, Ergotherapie, Sporttherapie und Rollstuhltraining angewendet werden. 


\section{Fehler, Gefahren, Komplikationen}

Die wesentlichste Gefahr der operativen Behandlung von posttraumatischen Fußinfektionen besteht darin, bei der Revision das wahre Ausmaß der Infektion nicht $\mathrm{zu}$ erkennen und dadurch $\mathrm{zu}$ wenig zu débridieren. Es empfiehlt sich dringend das mehrzeitige Vorgehen. Die Gefahr der externen Osteosynthese liegt darin, dass alleinige Schrauben am Fuß innerhalb weniger Wochen auslockern, dass Knochenschrauben häufig sekundär infizieren, speziell wenn die Patienten unerlaubt zu stark oder gar voll belasten. Auch muss daran gedacht werden, dass fixierende Elemente, also Knochenschrauben mit endständigem oder mittelständigem Gewinde, SteinmannNägel oder Titandrähte, aufgrund der komplizierten Anatomie falsch oder ungünstig platziert werden und dadurch Folgeprobleme auslösen.

\section{Folgeeingriffe}

In aller Regel sind nach eingetretener Infektberuhigung weitere Eingriffe zum Knochenaufbau und zum definitiven Weichteildefektverschluss erforderlich, wobei häufig der plastische Chirurg hinzugezogen werden muss. Auf die Probleme des Defektaufbaus wird hier nicht näher eingegangen.

\section{Allgemeine Nachbehandlung und Rehabilitationsmaßnahmen}

Nach Infektberuhigung wird zum frühestmöglichen Zeitpunkt mit der umfassenden Rehabilitation begonnen, was spätestens 5 Tage nach dem infektberuhigenden Eingriff möglich sein sollte. Nach Defektaufbau von Knochen und/ oder Weichteilen ist es nicht selten ratsam, eine Versorgung mit teilentlastendem Unterschenkelgehapparat vorzunehmen, um ein stockfreies Gehen $\mathrm{zu}$ erreichen.

\section{Berufliche Rehabilitations- maßnahmen}

Die meisten Fußinfektionen hinterlassen Schäden, sodass häufig Rehabilitationsmaßnahmen erforderlich werden. Meist ist eine Arbeits- und Belastungserprobung zur schrittweisen Rückführung in die alte Tätigkeit einzuleiten. Häufig können nach Behandlungsabschluss Arbeiten mit dauerndem Stehen und Gehen sowie das Arbeiten auf Leitern und Gerüsten nicht mehr wettbewerbsfähig ausgeführt werden, eine Umschulung wird erforderlich.

\section{Hilfsmittel}

Nach Fußinfektion kommt es regelmäßig zu kleineren oder stärkeren Schäden, weshalb zumindest eine Versorgung mit umfassend gewalkten Einlagen und/oder mit orthopädischem Maßschuhwerk erforderlich werden. Da die Belastungsfähigkeit nach ausgedehnteren Infektionen häufig erheblich eingeschränkt ist, ist es sinnvoll, die Unfallverletzten mit teilentlastendem Unterschenkelgehapparat oder teilentlastenden Unterschenkelkunststoffsandwichorthesen zu versorgen.

\section{Weiterführende Literatur}

1. EchtermeyerV (1991) Das Kompartmentsyndrom des Fußes. Orthopäde 20: 76-79

2. Khuri S, Ochsner PE (1986) Differentialdiagnose der Kalkaneusosteomyelitis.Z Orthop Ihre Grenzgeb 124: 19

3. Knopp W, Muhr G (1987) Wiederherstellungschirurgie bei posttraumatischen Weichteilschäden am Unterschenkel und Fuß. Unfallchirurg 90: 485-495

4. Müller KH (1981) Die exogene Osteomyelitis des Fußes. In: Müller KH (Hrsg) Exogene Osteomyelitis von Becken und unteren Gliedmaßen. Springer, Berlin Heidelberg New York, S 367

5. Roesgen M,Wytewicz A (1990) Posttraumatische Infektionen des Fußes. Schriftenreihe Unfallmed Tag Landesverband Gewerbl BG 74: 61-81

6. Schmidt HGK, Schultz J-H, Jürgens C, Wittek F (1995) Indikation und Technik der Amputation bei schweren Infektionen der Extremität. Hefte Unfallchir 255:212-230

7. Tscherne H (1986) Management der Verletzungen am distalen Unterschenkel und am Fuß. Langenbecks Arch Chir 369: 539-542

8. Winkel R (1994) Weichteilverletzungen des Fußes. Schriftenreihe Unfallmed Tag Landesverband Gewerbl BG 88:187-198

9. Zwipp H (1994) Chirurgie des Fußes. Springer, Berlin Heidelberg New York

10. Zwipp H, Tscherne H, Berger A (1989) Rekonstruktive Fußchirurgie nach Komplextraumen des Fußes. Unfallchirurg 92: 140-154 